\title{
On the Equivalence of the Two Most Favoured Calabi-Yau Compactifications
}

\author{
Brian R. Greene ${ }^{1 \star}$ and Kelley H. Kirklin ${ }^{2 \star \star}$ \\ 1 Lyman Laboratory of Physics, Harvard University, Cambridge, MA 02138, USA \\ 2 Department of Theoretical Physics, University of Oxford, 1 Keble Road. Oxford OX1 3NP, UK
}

\begin{abstract}
We discuss the two known multiply connected Calabi-Yau manifolds which give rise to three generations of elementary particles when chosen as the classical vacuum configuration of the $E_{8} \times E_{8}$ heterotic superstring. It is shown that these two manifolds are diffeomorphic.
\end{abstract}

\section{Introduction}

In addition to providing a potentially consistent unification of the fundamental forces, superstring theory $[1,2]$ has the appealing aspect of leading to highly constrained low energy models of particle interactions. Once a vacuum state is chosen much of the low energy structure of such models is determined. There is, of course, a catch [3]. One must first choose a vacuum configuration. Ideally, one hopes that further investigation of the underlying theory will ultimately show that the vacuum state is determined dynamically. For now, however, one does the next best thing by combining the demands of internal consistency and phenomenological viability. Analysis of this sort from a number of viewpoints has shown [4], that this favours vacuum configurations based upon Calabi-Yau compactifications; that is, vaccua of the form $M_{4} \times K$, where $M_{4}$ is four dimensional Minkowski space and $K$ is a three complex dimensional Kähler manifold with vanishing first Chern class. This certainly narrows down the choice, but, alas, there are many Calabi-Yau manifolds to choose from $[5,6]$. As noted, though, after a choice of the Calabi-Yau vacuum configuration $\mathrm{K}$ is made, much of the resulting low energy phenomenology may be extracted from the topological and cohomological properties of the manifold. In fact, in the case of the $E_{8} \times E_{8}$ Heterotic string, the number of generations of elementary particle multiplets (i.e. 27 's of $E_{6}$ ) is given by one half of the Euler characteristic of $K,|\chi(K) / 2|$ (after the $S U(3)$ spin connection $\omega$ of $K$

\footnotetext{
* Part of this work carried out at and supported by the IBM T.J. Watson Research Center, Yorktown Heights, NY

$\star \star$ On leave from Lyman Laboratory of Physics, Harvard University
} 
is identified with an $S U(3)$ subgroup of one of the $E_{8}$ factors of the Yang-Mills group), the unbroken gauge group in such a scenario depends upon the fundamental group $\pi_{1}(K)$ [7], and the Yukawa couplings upon certain products in the cohomology ring of $K[8,9]$.

Low energy phenomenology may thus be used to adjudicate amongst the many Calabi-Yau manifolds which a priori are equally viable vacuum configurations.

The easiest phenomenological constraint to impose is that of the number of generations; present experimental evidence indicates that Calabi-Yau manifolds with $|\chi(K)|=6$ are most favoured as they lead to three generations. A recent computer search $[10,11]$ for three generation Calabi-Yau manifolds realizable as smooth complete intersections in products of complex projective spaces modded out by freely acting projective discrete symmetry groups has shown that such manifolds are very rare. In fact, only one such manifold was found.

This manifold (originally constructed by Yau [5]), which we shall denote by $K_{1}$, is defined by the complete intersection in $C P^{3} \times C P^{3}$ of bidegree $(3,0)$, $(0,3)$ and $(1,1)$ homogeneous polynomials, modded out by a freely acting $Z_{3}$ discrete symmetry group. For definiteness, letting the four homogeneous coordinates of the first $C P^{3}$ space be denoted by $\left(X_{0}, \ldots, X_{3}\right)$ and similarly for the second with $X$ replaced by $Y, K_{1}$ is given by

$$
\left(\sum_{i=0}^{3} X_{i}^{3}=0\right) \times\left(\sum_{i=0}^{3} Y_{i}^{3}=0\right) \cap\left(\sum_{i=0}^{3} X_{i} Y_{i}=0\right)
$$

modded out by the $Z_{3}$ group $G$ generated by the map $g$ :

$$
g:\left(X_{0}, X_{1}, X_{2}, X_{3}, Y_{0}, Y_{1}, Y_{2}, Y_{3}\right) \rightarrow\left(X_{0}, \alpha^{2} X_{1}, \alpha X_{2}, \alpha X_{3}, Y_{0}, \alpha Y_{1}, \alpha^{2} Y_{2}, \alpha^{2} Y_{3}\right) \text {, }
$$

where $\alpha$ is a nontrivial cube root of unity. (The defining equations (1.1) correspond to a particular choice of the complex structure for $K_{1}$; varying these equations in a $G$-invariant manner yields a space which if nonsingular, is diffeomorphic to $K_{1}$.)

This manifold, has been shown to yield remarkably realistic phenomenology when chosen for the vacuum configuration [12]. In addition to $K_{1}$, two other Calabi-Yau manifolds with $|\chi|=6$ are known to exist, both constructed by Yau [5]. One of these is simply connected and hence is not amenable to the flux trapping method of gauge symmetry breaking, making it less phenomenologically promising. The other, which we denote by $K_{2}$ is as follows [5].

$$
K_{2}=\overparen{K_{0}^{\prime} / G_{27}} \text {, }
$$

where $K_{0}^{\prime}$ is a bidegree $(3,3)$ hypersurface in $C P^{2} \times C P^{2}, G_{27}$ is a non-freely acting symmetry group of order 27 , and the tilde denotes that the resulting fixed points are resolved.

At present therefore, detailed search for Calabi-Yau manifolds which give rise to three generations has provided two multiply connected (and hence of phenomenological interest) possibilities. In this note we show that these 'two' possibilities are actually diffeomorphic, with the three generation model analysed in [12] giving the relevant phenomenology for a particular choice of the vacuum moduli. 


\section{Fundamental Group and Hodge Diamond}

At first sight this claim seems unlikely as the Hodge diamonds and fundamental groups of these two manifolds have been reported as being distinct [5]. We now show that they are in fact identical.

Recall from [5] and that fundamental group of $K_{1}$ is $Z_{3}$ and from [12] that the Hodge diamond for $K_{1}$ and its covering space $K_{0}$ are given by:

$$
\begin{array}{cccccccc} 
& & & 1 & & & \\
& & & 0 & & 0 & & \\
& & 0 & & 6 & & 0 & \\
& & 9 & & 9 & & 1 \\
& 0 & & 6 & & 0 & \\
& & 0 & & 0 & & \\
& & & & 1 & & & \\
& & & & & & &
\end{array}
$$

and

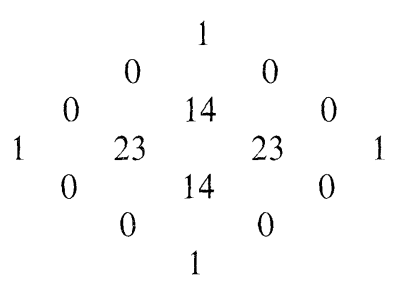

respectively.

To construct $K_{2}$ we choose the nonsingular defining polynomial for the bicubic $K_{0}^{\prime}$ to be

$$
\sum_{i=0}^{2} x_{i}^{3} y_{i}^{3}+\delta\left(\sum_{i=0}^{2}\left(x_{i}^{3} y_{i+1}^{3}+x_{i}^{3} y_{i-1}^{3}\right)\right)=0,
$$

where $\delta$ is a complex number, the x's and y's are homogeneous $C P^{2}$ coordinates $^{1}$, and we mod through by the (non-freely acting) group $G_{27}$ generated by the three maps:

$$
\begin{gathered}
\sigma_{1}:\left(x_{0}, x_{1}, x_{2}\right) \times\left(y_{0}, y_{1}, y_{2}\right) \rightarrow\left(x_{1}, x_{2}, x_{0}\right) \times\left(y_{1}, y_{2}, y_{0}\right), \\
\sigma_{2}:\left(x_{0}, x_{1}, x_{2}\right) \times\left(y_{0}, y_{1}, y_{2}\right) \rightarrow\left(x_{0}, \alpha x_{1}, \alpha^{2} x_{2}\right) \times\left(y_{0}, \alpha y_{1}, \alpha^{2} y_{2}\right), \\
\sigma_{3}:\left(x_{0}, x_{1}, x_{2}\right) \times\left(y_{0}, y_{1}, y_{2}\right) \rightarrow\left(x_{0}, x_{1}, x_{2}\right) \times\left(y_{0}, \alpha y_{1}, \alpha^{2} y_{2}\right) .
\end{gathered}
$$

Notice that $\sigma_{3}$ and $\sigma_{2}^{2} \sigma_{3}$ each have three fixed tori when acting on the manifold $K_{0}^{\prime}[5]$.

To compute the Hodge diamond, we proceed in stages. On $K_{0}^{\prime}$ we apply the Lefschetz Theorem in the form

$$
h^{p . q}\left(K_{0}^{\prime}\right)=h^{p, q}\left(C P^{2} \times C P^{2}\right) \text { for } p+q<3
$$

to conclude that $h^{1,1}=2, h^{1,0}=h^{0,1}=0$, and of course, $h^{0,0}=1$. From the adjunction formula [13] we have $\chi\left(K_{0}^{\prime}\right)=-162$, and hence $h^{1,1}-h^{1,2}=-81$.

1 Throughout this letter, $C P^{2}$ homogeneous coordinates are given by lowercase letters, and $C P^{3}$ homogeneous coordinates are given by uppercase letters 
Thus, we have the Hodge Diamond:

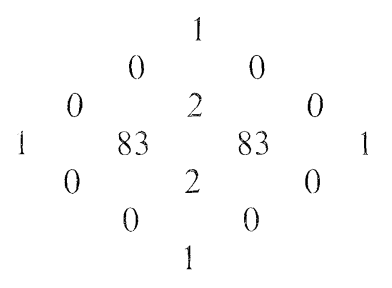

The group generated by $\sigma_{1}$ and $\sigma_{2}$ does act freely and hence $K_{0}^{\prime} /\left(\sigma_{1}, \sigma_{2}\right)$ is smooth and has Euler characteristic 18 . Also, $h^{1.1}$ remains two, as these are just the pullbacks of the two $C P^{2} \mathrm{Kähler}$ forms which are invariant under $\sigma_{1}$ and $\sigma_{2}$. Thus, $h^{1.2}$ on this quotient space is 11 .

The final step in the construction is modding out by $\sigma_{3}$. The easiest way to analyse the resulting cohomology is to split it into a sum of that derived from the non-singular part of the space with that derived from blowing up the singular tori.

For the nonsingular part, $V$, we employ the same reasoning as above to write the contribution to the Hodge Diamond of $K_{2}$ as:

$\begin{array}{cccccccc} & & & 1 & & & \\ & & & 0 & & 0 & & \\ & & 0 & & 2 & & 0 & \\ & & 5 & & 5 & & 1 \\ & 0 & & 2 & & 0 & \\ & & 0 & & 0 & & \\ & & & & 1 & & & \end{array}$

The value of $\chi(V)$ is -6 .

To analyse the changes in the cohomology which result from blowing up the singularities, we first study these singularities locally. Around any will-be fixed point on $\mathrm{V}$, we may construct a $B^{4}$ (the four ball) with boundary being an $S^{3}$. On modding out by $\sigma_{3}$, this $S^{3}$ becomes the Lens space $L(3,2)$. Thus, to resolve a single fixed point (on any of the singular tori) we must 'glue' in a smooth four space $Q^{4}$ with $\partial Q^{4}=L(3,2)$.

The construction of such a $Q^{4}$ is a well studied problem and has been solved for the general $L(p, q)$ scenario [14]. In this case, we must take $Q^{4}$ to be the plum product of two copies of $S^{2}(-2)$, where $S^{2}(-2)$ is the disk bundle over $S^{2}$ with first Chern number equal to -2 . (In singularity theory resolving spaces of this type are known as $\left(A_{2}\right)$ Hirzebruch-Jung strings [15].)

To address the full problem of blowing up the fixed tori, we must examine the structure of their respective normal bundles in order to understand how they are embedded in the ambient threefold. Consider one such torus (the other five are found by cyclically permuting these coordinates and swapping $\mathrm{x}$ and $\mathrm{y}$ coordinates):

$$
\left(x_{0}^{3}+\delta\left(x_{1}^{3}+x_{2}^{3}\right)=0\right) \times(1,0,0) .
$$

At any point on this torus, we may take $\left(\zeta_{1}, \zeta_{2}\right)$, where $\zeta_{i}=y_{i} / y_{0}$ as suitable normal bundle coordinates, giving us a global frame. The triviality of the normal bundle allows us to extend the resolution procedure described above merely by 
extracting a tubular neighborhood around any of the fixed tori of the form

$$
T^{2} \times B^{4}
$$

on $K_{0}^{\prime}$, and replacing it by

$$
T^{2} \times Q^{4}
$$

on $K_{2}$. Viewed on $K_{2}$, this procedure extracts $T^{2} \times C L(3,2)$ (where $C L(3,2)$ is the cone over $L(3,2))$ and replaces it by $T^{2} \times Q^{4}$. Since the Euler characteristic is multiplicative, we see that this procedure preserves the $\chi\left(K_{2}\right)=-6$.

However, the individual Hodge numbers are not preserved. The Hodge diamond of $T^{2}$ is simply:

$$
11
$$

While, similarly, the Hodge diamond of $T^{2} \times Q^{4}$ is that of $T^{2} \times\left(S^{2} \wedge S^{2}\right)$ (where the $\wedge$ denotes the plum product, and equality follows because the latter is the deformation retract of $T^{2} \times Q^{4}$ ). This gives Hodge diamond for $T^{2} \times Q^{4}$ :

$$
\begin{array}{cccccccc} 
& & & 1 & & & & \\
& & 0 & & 3 & & 0 & \\
0 & & 2 & & 2 & & 0 \\
& 0 & & 2 & & 0 & \\
& & 0 & & 0 & & \\
& & & 1 & & &
\end{array}
$$

On $K_{2}$ we must perform this procedure twice (for each of the two pairs of three fixed tori). This yields the net change in the Hodge diamond being:

$$
\begin{array}{cccccccc} 
& & & & 0 & & & \\
& & 0 & & 0 & & \\
& 0 & & 4 & & 0 & \\
& & 4 & & 4 & & 0 \\
& 0 & & 4 & & 0 & \\
& & 0 & & 0 & & \\
& & & 0 & & &
\end{array}
$$

Finally, combining this result with that for the non-singular part $V$ of $K_{2}$ we find that the resolved Hodge diamond is:

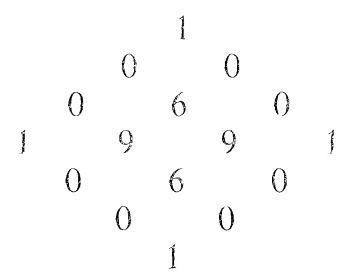

which is precisely that of $K_{1}$. Furthermore, following from the above, the preimage under $\sigma_{1}$ of each of the resolved tori gives a three sheeted cover, as $\sigma_{1}$ permutes 
the tori in two groups of three. Diagonalizing this $Z_{3}$ we thus see that the resolution contributes $12(2,1)$ and $12(1,1)$ forms which each comprise three regular representations of $Z_{3}$. Thus, we see that a covering space for $K_{2}$ has the Hodge diamond:

$\begin{array}{cccccccc} & & & 1 & & & \\ & & & 0 & & 0 & & \\ & 0 & & 14 & & 0 & \\ & & 23 & & 23 & & 1 \\ & 0 & & 14 & & 0 & \\ & & 0 & & 0 & & \\ & & & 1 & & & \end{array}$

Notice that this is precisely that of $K_{0}$. One might fear that this resolution procedure may have effected the vanishing of the first Chern class and Kählerity properties manifest for $K_{0}^{\prime}$; it can be shown however that $K_{2}$ inherits both of these characteristics [15].

To address the identity of the fundamental group on the blown up threefold, it is simplest to reverse the order of the original construction. Starting from the manifold $K_{0}^{\prime}$ as given in (2.3), we mod through by the group generated by $\sigma_{2}$ and $\sigma_{3}$. As noted, not only does $\sigma_{3}$ have three fixed tori, but $\sigma_{2}^{2} \sigma_{3}$ has three fixed tori as well. These codimension two fixed point sets then allow us to contract any would-be nontrivial loop associated with the passage to the quotient space; i.e. $K_{0}^{\prime} /\left(\sigma_{2}, \sigma_{3}\right)$ is simply connected. Upon resolving the fixed tori using the procedure described above, we then arrive at a smooth simply connected manifold, $K_{0}^{\prime \prime}$, with Euler characteristic 18, this is the covering space of $K_{2}$. The final step is then to mod through by $\sigma_{1}$. As this transformation acts freely, when extended to permute the exceptional divisors of the toroidal blow-up's rather than the singular tori themselves, it gives rise to a fundamental group of $Z_{3}$ on the quotient manifold $K_{2}$.

The above arguments make our claim that $K_{1}$ and $K_{2}$ are diffeomorphic a strong possibility. Before making the diffeomorphism explicit, we note the intuitive identification we shall make.

First recall the standard result from algebraic geometry that a cubic hypersurface in $C P^{3}$ may be realized as a $C P^{2}$ with six points blown up [16]. Then notice that if the $C P^{3}$ coordinates are chosen to be cubic functions of the $C P^{2}$ coordinates, the bicubic equation in $K_{2}$ is mapped to a bilinear equation, i.e. a bidegree $(1,1)$ relation. Apparently then, the two $C P^{3}$ cubic surfaces which are part of the definition of $K_{1}$ may arise from the ambient $C P^{2}$ spaces (via the resolution of singularities), and the $(1,1)$ hyperplane arises as the image of the bicubic defining equation. We make this intuitive notion explicit in the next section.

\section{Explicit Diffeomorphism}

Thus far we have shown that the two $\chi=-6$ manifolds $K_{1}$ and $K_{2}$ have precisely the same Hodge diamond and fundamental group. This suggests quite strongly, but does not prove, that $K_{1}$ and $K_{2}$ are diffeomorphic. Before outlining a proof that this is indeed the case, we must discuss a few results from the theory of complex surfaces $[15,17,18,14]$. 
Consider a relation, $f$, from $C P^{2}$ to $C P^{3}$ given by

$$
f:\left(x_{0}, x_{1}, x_{2}\right) \rightarrow\left(x_{0}^{3}, x_{1}^{3}, x_{2}^{3}, x_{0} x_{1} x_{2}\right)=\left(X_{0}, X_{1}, X_{2}, X_{3}\right) .
$$

This relation is three-to-one except at the three $C P^{2}$ points $(0,0,1),(0,1,0)$, and $(1,0,0)$ where it is one-to-one. The image in $C P^{3}$ is the singular cubic surface defined by

$$
X_{3}^{3}-X_{0} X_{1} X_{2}=0 \text {. }
$$

This surface is singular at the three $C P^{3}$ points $(0,0,1,0),(0,1,0,0)$, and $(1,0,0,0)$ which are precisely the images of the three one-to-one points of $f$. Now if we mod through by the nonfreely acting $Z_{3}$ on $C P^{2}$ generated by

$$
h:\left(x_{0}, x_{1}, x_{2}\right) \rightarrow\left(x_{0}, \alpha x_{1}, \alpha^{2} x_{2}\right)
$$

we get a homeomorphism $\tilde{f}$ between singular spaces from $f$ in the obvious way. (The action of h makes the map one-to-one everywhere on $C P^{2} / Z_{3}$.)

$$
\tilde{f}: C P^{2} / Z_{3} \rightarrow\left(X_{3}^{3}-X_{0} X_{1} X_{2}=0\right) \subset C P^{3} .
$$

If we blow up the three singular points on either side of the homeomorphism $\tilde{f}$, we get a diffeomorphism between Kähler manifolds. The relevant blow-up procedure for each of the points involves an $A_{2}$ Hirzebruch-Jung string (as discussed above this is a certain disk bundle over the plumb product of two $S^{2}$ 's) [14].

It is a important result of the theory of the resolution of rational singularities of complex surfaces that the resolution of the singular points of $\left(X_{3}^{3}-X_{0} X_{1} X_{2}=0\right) \subset C P^{3}$ yields a surface which is diffeomorphic to the generic nonsingular cubic surface in $C P^{3}[19]$. This result and the above discussion imply:

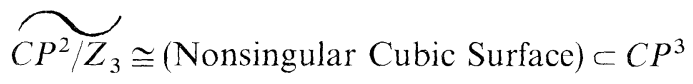

(where the $Z_{3}$ is generated by the map $h$ above and the tilde denotes resolution).

Equation (3.5) is one form of the fact that the cubic surface is diffeomorphic to the $C P^{2}$ with six (nonsingular) points blown-up [16]. (We can move around the six exceptional divisors differentially, and (3.5) depends roughly on them coinciding in three pairs.)

We can apply these facts to show that the covering spaces of $K_{1}$ and $K_{2}$ are diffeomorphic. To this end it is convenient to slightly deform the $C P^{2} \times C P^{2}$ bicubic polynomial from which $K_{2}$ is constructed to the form:

$$
B \cong\left(\sum_{i=0}^{2} x_{i}^{3} y_{i}^{3}+x_{0} x_{1} x_{2} y_{0} y_{1} y_{2}+\delta\left(\sum_{i=0}^{2}\left(x_{i}^{3} y_{i+1}^{3}+x_{i}^{3} y_{i-1}^{3}\right)\right)=0\right) \subset C P^{2} \times C P^{2} .
$$

(This change does not effect the construction of $K_{2}$; it corresponds to a slight change in moduli. To prove $K_{1}$ and $K_{2}$ are diffeomorphic it suffices to show that any two nonsingular points in their respective moduli spaces are diffeomorphic.)

As discussed above the covering space of $K_{2}$ is constructed by resolving the singular curves of a $C P^{2} \times C P^{2}$ bicubic which are introduced by modding out the 
nonfreely acting group generated by

and

$$
\sigma_{2}^{2} \sigma_{3}:\left(x_{0}, x_{1}, x_{2}\right) \times\left(y_{0}, y_{1}, y_{2}\right) \rightarrow\left(x_{0}, \alpha x_{1}, \alpha^{2} x_{2}\right) \times\left(y_{0}, y_{1}, y_{2}\right)
$$

$$
\sigma_{3}:\left(x_{0}, x_{1}, x_{2}\right) \times\left(y_{0}, y_{1}, y_{2}\right) \rightarrow\left(x_{0}, x_{1}, x_{2}\right) \times\left(y_{0}, \alpha y_{1}, x^{2} y_{2}\right) .
$$

We write the two generators in this way to exploit the fact that they have precisely the same form as $h$ defined in (3.3) above; $\sigma_{2}^{2} \sigma_{3}$ acts on the left $C P^{2}$ and $\sigma_{3}$ on the right $C P^{2}$. If we ignore the bicubic equation for the moment, the discussion above gives the homeomorphism:

$$
F:\left(C P^{2} \times C P^{2}\right) /\left(\sigma_{2}, \sigma_{3}\right) \rightarrow\left(X_{3}^{3}-X_{0} X_{1} X_{2}=0\right) \times\left(Y_{3}^{3}-Y_{0} Y_{1} Y_{2}=0\right) \subset C P^{3} \times C P^{3}
$$

with

$$
\begin{aligned}
F:\left(x_{0}, x_{1}, x_{2}\right) \times\left(y_{0}, y_{1}, y_{2}\right) & \mapsto\left(x_{0}^{3}, x_{1}^{3}, x_{2}^{3}, x_{0} x_{1} x_{2}\right) \times\left(y_{0}^{3}, y_{1}^{3}, y_{2}^{3}, y_{0} y_{1} y_{2}\right) \\
& =\left(X_{0}, X_{1}, X_{2}, X_{3}\right) \times\left(Y_{0}, Y_{1}, Y_{2}, Y_{3}\right) .
\end{aligned}
$$

In addition note that the map $F$ carries the bicubic equation to a bidegree $(1,1)$ polynomial in $C P^{3} \times C P^{3}$. Thus as singular spaces,

$$
\begin{aligned}
B /\left(\sigma_{2}, \sigma_{3}\right) & \cong\left(X_{3}^{3}-X_{0} X_{1} X_{2}=0\right) \times\left(Y_{3}^{3}-Y_{0} Y_{1} Y_{2}=0\right) \\
& \cap\left(\sum_{i=0}^{3} X_{i} Y_{\imath}+\delta\left(X_{0} Y_{1}+X_{1} Y_{2}+X_{2} Y_{0}+X_{0} Y_{2}+X_{1} Y_{0}+X_{2} Y_{1}=0\right)\right) \\
& \subset C P^{3} \times C P^{3}
\end{aligned}
$$

Thus, before resolution of the six singular tori to yield the covering space of $K_{2}$, the singular space $B /\left(\sigma_{2}, \sigma_{3}\right)$ is homeomorphic to a 'singular point' in the moduli space of bidegree $(3,0),(0,3),(1,1)$ complete intersections in $C P^{3} \times C P^{3}$. Generic nonsingular points of this moduli space correspond to the covering space of $K_{1}$. Thus to show that the two covering spaces are diffeomorphic, we need to show that resolving the codimension two singularities of the space (3.11) yields a manifold diffeomorphic to a nonsingular manifold with the same degree defining polynomials. (In one lower dimension, this would be analogous to the property of rational surface singularities discussed above.) In general this is not the case for three complex dimensional algebraic varieties [20]. However, in the case (3.11) at hand, we can resolve three of the singular tori by an $A_{2}$ resolution of the three singular points of the $Y$-space cubic $\left(Y_{3}^{3}-Y_{0} Y_{1} Y_{2}=0\right)$ thought of as a surface in $C P^{3}$. (We can think of the $Y_{i}$ as indexing a family of hyperplane sections of this cubic via the bidegree $(1,1)$ polynomial. The locus where these hyperplanes meet the singular points of the X-space cubic sweeps out three singular tori in the complete intersection. Thus resolving the $\mathrm{Y}$-space $C P^{3}$ cubic singular points in this manner is equivalent to resolving the singular torus given in (2.10) (along with its two partners obtained by cyclically permuting the coordinates).

We can clearly repeat this procedure for the $X$-space cubic and thus the theorems of [19] imply that the resolution of the singular curves in (3.11) yields a manifold which is diffeomorphic to the generic nonsingular bidegree $(3,0),(0,3),(1,1)$ complete intersection in $C P^{3} \times C P^{3}$. This completes the proof that the covering spaces of $K_{1}$ and $K_{2}$ are diffeomorphic. 
To extend this proof to $K_{1}$ and $K_{2}$ themselves we must consider the nature of the freely acting $Z_{3}$ actions relevant to each space. As discussed earlier, the map $\sigma_{1}$ of (2.4) is readily extended to an action on the smooth covering space $K_{0}^{\prime \prime}$ of $K_{2}$; under the map $F$ this extension is carried to the transformation $g$ :

$$
g:\left(X_{0}, X_{1}, X_{2}, X_{3}\right) \times\left(Y_{0}, Y_{1}, Y_{2}, Y_{3}\right) \rightarrow\left(X_{1}, X_{2}, X_{0}, X_{3}\right) \times\left(Y_{1}, Y_{2}, Y_{0}, Y_{3}\right)
$$

in $C P^{3} \times C P^{3}$. This $Z_{3}$ is in turn related by a PGL symmetry of the ambient $C P^{3} \times C P^{3}$ to the one employed in [12]. (From another point of view, a basis can be chosen for the exceptional divisors of $K_{1}^{\prime} s$ covering space in which the action of the free $Z_{3}$ is identical to that of $\sigma_{1}$ above [21].) More details concerning the equivalence of these $Z_{3}^{\prime} s$ shall be presented in [10].

\section{Phenomenological Implications}

As noted earlier, low energy superstring phenomenology, to a large extent, is determined by the manifold of compactification. Beyond the (topological) Hodge numbers which determine the number of generations and antigenerations of elementary particle multiplets, the Yukawa couplings follow from quasitopological computations in the cohomology ring of the chosen manifold. For negative Euler characteristic Calabi-Yau manifolds (such as those discussed in this letter) the Yukawa couplings amongst the antigenerations (the $(1,1)$ forms) are independent of the complex structure imposed on the manifold, while those amongst the generations (the $(2,1)$ forms) certainly are dependent on the complex structure [8]. One diffeomorphism class of Calabi-Yau manifolds thus has the potential to give rise to many possible low energy models. Our result, therefore, does not preclude the possibility of building phenomenologically interesting models based on this diffeomorphism class of compactifications leading to three generations other than that presented in [12], but shows that any such models will differ from that of [12] merely by a change in the choice of the complex structure.

Acknowledgements. We would like to thank S. Donaldson, N. Hitchin, J. Morgan and T. Phillips for valuable discussions. We also acknowledge useful discussions with P. Aspinwall, P. Miron and J. Distler. BRG is partially supported by NSF contract PHY-82-15249 and he gratefully thanks J. Cocke, G. Lasher and the Physical Sciences department of the IBM Watson research center for hospitality and financial support. KHK acknowledges the support of an NSF graduate fellowship.

\section{Note added in proof}

After the completion of this work, we received a preprint from R. Schimmrigk [22] presenting a third construction of a $\chi=-6$ Calabi-Yau manifold with $h^{1}=6, h^{2,1}=9$, and $\pi_{1} \cong Z$. This manifold is built from a bidegree $(3,0),(1,3)$ complete intersection in $C P^{3} \times C P^{2}$ by resolving the fixed curves of a $Z_{3}$ similar to that generated by $h$ in (3.3) above, and then modding out a freely acting $Z_{3}$. The discussions of this letter can easily be shown to imply that this third manifold is diffeomorphic to $K_{1}$ and $K_{2}$.

\section{References}

1. Schwarz, J. H.: Superstrings. Singapore: World Scientific 1986

2. Green, M. B. Schwarz, J. H. Witten, E.: Superstring theory. Cambridge. Cambridge Unıv. Press 1987 
3. Heller, J.: Catch-22, New York: Simon and Schuster 1961

4. Candelas, P. Horowitz, G. Stromınger, A. Witten, E.: Nucl. Phys. B258, (1985)

5. Yau, S.-T.: Argonne symposium on anomalies, geometry and topology. Singapore: World Scientific 1985

6. Hübsch, T.: University of Maryland preprint (1986)

7. Witten, E.: Nucl. Phys. B258, 75 (1985)

8. Strominger, A. Witten, E.: Commun Math. Phys. 101, 341 (1985)

9. Strominger, A.: Phys. Rev. Lett. 55, 2547 (1985) and in: Unified String theories. Green M. Gross, D. (eds.), Singapore: World Scientific 1986

10. Aspinwall, P. Greene, B. R. Kirklin K. H. Miron, P. J.: Searching for three-generation Calabi--Yau manifolds, Oxford preprint

11. Candelas, P.: in preparation

12. Greene, B. R. Kirklin, K. H. Mıron, P. J. Ross, G. G.: Nucl. Phys. B278, 667 (1986), Phys. Lett. 180B, 117 (1986) and Oxford preprint 11/87

13. Griffths, P. Harris, J.: Principles of algebraic geometry. New York: Wiley 1978

14. Brieskorn,: Die Ausflosung der Rationalen Singularitaten Holomorpher Abbildungen. Math. Ann. 178 (1968)

15. Barth, W., Peters, C., van de Ven, A.: Compact complex surfaces. Berlin. Heidelberg, New York: Springer 1984

16. See Hartshorne, R.: Algebraic geometry. Berlin, Heidelberg, New York: Springer 1977 for a full discussion

17. Hitchen, N.: Polygons and gravitons. Math. Proc. Camb. Phil. Soc. 85 (1979)

18. Milnor, J.: Singular points of complex hypersurfaces. Princeton, NJ: Princeton Univ. Press 1968

19. Atiyah, M.: On Analytic surfaces with double points. Proc. Roy. Soc. London Ser. A247, (1958)

20. Hitchin, N.: private communication

21. Distler, J. Greene, B. Kirklin, K. Miron, P.: (to appear)

22. Schimmrigk, R.: Texas preprint

Communicated by A. Jaffe

Received March 27, 1987 